\title{
ALL AUTOMORPHISMS OF ALL CALKIN ALGEBRAS
}

\author{
ILIJAS FARAH
}

\begin{abstract}
The Proper Forcing Axiom implies all automorphisms of every Calkin algebra associated with an infinite-dimensional complex Hilbert space and the ideal of compact operators are inner. As a means of the proof we introduce notions of metric $\omega_{1}$-trees and coherent families of Polish spaces and develop their theory parallel to the classical theory of trees of height $\omega_{1}$ and coherent families indexed by a $\sigma$-directed ordering.
\end{abstract}

Fix an infinite-dimensional complex Hilbert space $H$. Let $\mathcal{B}(H)$ be its algebra of bounded linear operators, $\mathcal{K}(H)$ its ideal of compact operators and $\mathcal{C}(H)=$ $\mathcal{B}(H) / \mathcal{K}(H)$ the Calkin algebra. Answering a question first asked by Brown-DouglasFillmore, in [10] and [5] it was proved that the existence of outer automorphisms of the Calkin algebra associated with a separable $H$ is independent from ZFC. In the present paper we consider the existence of outer automorphisms of the Calkin algebra associated with an arbitrary complex, infinite-dimensional Hilbert space.

PFA stands for the Proper Forcing Axiom, MA for Martin's Axiom and TA stands for Todorcevic's Axiom (see e.g., [12] or [8] for PFA and TA and [7, Chapter II] for MA). It is well-known that both MA and TA are consequences of PFA.

Theorem 1. MA and TA together imply all automorphisms of the Calkin algebra associated with Hilbert space with basis of cardinality $\aleph_{1}$ are inner.

Theorem 2. PFA implies all automorphisms of every Calkin algebra are inner.

The only use of TA in the present paper is implicit via the following result from [5].

Theorem 3. TA implies all automorphisms of the Calkin algebra on a separable, infinite-dimensional Hilbert space are inner.

All of these results are part of the program of finding set-theoretic rigidity results for algebraic quotient structures. This program can be traced back to Shelah's seminal construction of a model of ZFC in which all automorphisms of $\mathcal{P}(\mathbb{N}) /$ Fin are trivial ([11]). At present we have a non-unified collection of results and it is unclear how far-reaching this phenomenon is (see [2, §3.2], [3], [4] and the last section of [5]).

The rudimentary idea of the proofs of Theorem 1 and Theorem 2 is taken from the analogous Velickovic's results on automorphisms of the Boolean algebra $\mathcal{P}(\kappa) /$ Fin in $[13, \S 4]$. A sketch of Velickovic's argument is in order for the reader's benefit.

If $\Phi$ is an automorphism of $\mathcal{P}\left(\omega_{1}\right) /$ Fin then there is a closed unbounded set $C \subseteq \omega_{1}$ such that for every $\alpha \in C$ the restriction of $\Phi$ to $\mathcal{P}(\alpha) /$ Fin is an automorphism of $\mathcal{P}(\alpha) /$ Fin. Since MA and TA imply that all automorphisms of $\mathcal{P}(\omega) /$ Fin are trivial ([13, Theorem 2.1]), for each $\alpha \in C$ we can fix a map $h_{\alpha}: \alpha \rightarrow \alpha$ such that the map $\mathcal{P}(\alpha) \ni A \mapsto h_{\alpha}[A] \in \mathcal{P}(\alpha)$ is a representation of the restriction of $\Phi$ to $\mathcal{P}(\alpha) /$ Fin.

Received by the editors July 23, 2010. 
For $\alpha<\beta<\gamma$ with $\beta$ and $\gamma$ in $C$ we have that $h_{\beta}\left\lceil\alpha\right.$ and $h_{\gamma} \uparrow \alpha$ agree modulo finite. Therefore

$$
T=\left\{h_{\beta} \uparrow \alpha: \alpha<\beta, \beta \in C\right\},
$$

considered as a tree with respect to the extension ordering, has countable levels. Automorphism $\Phi$ is trivial if and only if $T$ has a cofinal branch. For every $f: \omega_{1} \rightarrow 2$ the tree

$$
T[f]=\{f \circ t: t \in T\}
$$

has a cofinal branch, determined by $Y$ such that $[Y]_{\text {Fin }}=\Phi\left([X]_{\text {Fin }}\right)$, where $f=\chi_{X}$. On the other hand, if $\dot{f}$ is added by forcing with finite conditions $\mathbb{P}$ (i.e., if $\dot{f}$ codes a set of $\aleph_{1}$ side-by-side Cohen reals over $V$ ) then $\mathbb{P}$ forces that $T[\dot{f}]$ has no cofinal branches. Applying MA to the poset for adding $\dot{f}$ followed by the ccc poset for specializing $T[\dot{f}]$ one obtains a contradiction.

Velickovic's proof of triviality of automorphisms of $\mathcal{P}(\kappa) /$ Fin for $\kappa \geq \aleph_{2}$ uses a PFA-reflection argument, in which the above proof is preceded by a Levy collapse of $\kappa$ to $\aleph_{1}$.

While the structure of our proof of Theorem 1 loosely resembles the above sketch, a number of nontrivial additions and modifications were required. For example, it is not clear whether for every automorphism $\Phi$ of $\mathcal{C}\left(\ell_{2}\left(\aleph_{1}\right)\right)$ the set $C$ of countable ordinals $\alpha$ such that the restriction of $\Phi$ to $\mathcal{C}\left(\ell_{2}(\alpha)\right)$ is an automorphism of the latter algebra is closed and unbounded. This follows from MA+TA by Theorem 1, but I don't know whether this fact is true in ZFC. This problem is dealt with in $\S 3.1$. An another inconvenience was caused by the fact that the natural 'quantized' analogue of the poset for adding $\aleph_{1}$ Cohen reals is not ccc (Lemma 4.1), as well as the expected non-commutativity complications.

Also, the appropriate analogues of Velickovic's trees $T$ and $T[f]$ are continuous rather than discrete. Therefore the proof of Theorem 1 required introduction and analysis of 'metric $\omega_{1}$-trees,' analogous to the classical theory of $\omega_{1}$-trees. This was done in $\S 1$. This section is independent of the rest of the paper and it is 'purely set-theoretic' in the sense that $\mathrm{C}^{*}$-algebras are not being mentioned in it.

The structure of the paper. Metric $\omega_{1}$-trees and metric coherent families are introduced and treated using MA and PFA, respectively, in $\S 1$. The short $\S 2$ contains a few simple and well-known general facts about inner automorphisms of $\mathrm{C}^{*}$-algebras. In $\S 3$ we define analogues of trees $T$ and $T[f]$ from Velickovic's proof, and in $\S 4$ we analyze $T[\tau]$ for an appropriately defined generic operator $\tau$. Proof of Theorem 2 and brief concluding remarks can be found in $\S 5$ and $\S 6$, respectively.

Our notation and terminology are standard and excellent references for the background on $\mathrm{C}^{*}$-algebras and set theory are [1] and [7], respectively. Introductions to applications of combinatorial set theory to $\mathrm{C}^{*}$-algebras can be found in [14] and [6].

\section{Acknowledgments}

Partially supported by NSERC. This work started in conversations with Ernest Schimmerling and Paul McKenney while we visited the Mittag-Leffler Institute in September 2009. I would like to thank the staff of the Institute for providing a pleasant and stimulating atmosphere and the anonymous referee for several useful suggestions. This proof was first presented in a minicourse during the 2009 workshop 
on Combinatorial set theory and forcing theory at the Research Institute in Mathematics in Kyoto. I would like to thank the organizer of the workshop, Teruyuki Yorioka, for his warm hospitality.

\section{Polish $\omega_{1}$-trees}

In this section we introduce a continuous version of Aronszajn trees. A note on terminology is in order. In operator algebras 'contraction' commonly refers to a map that is distance-non-increasing. In some other areas of mathematics such maps are referred to as 1-Lipshitz and 'contraction' refers to a distance-decreasing map. The latter type of a map is referred to as a strict contraction by operator algebraists. In what follows I use the operator-algebraic terminology, hence a contraction $f$ is assumed to satisfy $d(x, y) \geq d(f(x), f(y))$. Other than this concession, the theory of operator algebras does not make appearance in the present section.

A metric $\omega_{1}$-tree is a family $T=\left(X_{\alpha}, d_{\alpha}, \pi_{\beta \alpha}\right.$, for $\left.\alpha \leq \beta<\omega_{1}\right)$, such that

(1) $X_{\alpha}$ is a complete metric space with compatible metric $d_{\alpha}$,

(2) $\pi_{\beta \alpha}: X_{\beta} \rightarrow X_{\alpha}$ is a contractive surjection,

(3) projections $\pi_{\beta \alpha}$ are commuting and $\pi_{\alpha \alpha}=\mathrm{id}_{X_{\alpha}}$ for all $\alpha$.

If all spaces $X_{\alpha}$ are separable we say $T$ is a Polish $\omega_{1}$-tree. If in addition the inverse limit $\lim _{\alpha} X_{\alpha}$ is empty then we say that $T$ is a Polish Aronszajn tree. Otherwise, the elements of the inverse limit $\lim _{\alpha} X_{\alpha}$ are considered to be branches through $T$. In our terminology all branches and all $\varepsilon$-branches are assumed to be cofinal.

When each $d_{\alpha}$ is a discrete metric then the above definitions reduce to the usual definitions of $\omega_{1}$-trees and Aronszajn trees (see e.g., [7]). Similarly, $\varepsilon$-branches, $\varepsilon$ antichains and $\varepsilon$-special trees as defined below are branches, antichains, and special trees, respectively, when $0<\varepsilon<1$.

Spaces $X_{\alpha}$ are assumed to be disjoint and we shall identify $T$ with the union $\bigcup_{\alpha} X_{\alpha}$ of its levels when convenient and the projections are clear from the context. On $T$ we have a map Lev: $T \rightarrow \omega_{1}$ defined by $\operatorname{Lev}(x)=\alpha$ if and only if $x \in X_{\alpha}$.

It will be convenient to write $\pi_{\alpha}$ for the map $\bigcup_{\beta \geq \alpha} \pi_{\beta, \alpha}$ from $T$ into $T_{\alpha}$. Define a map $\rho$ on $T^{2}$ as follows. For $x, y$ in $T$ let $\alpha=\min (\operatorname{Lev}(x), \operatorname{Lev}(y))$ and let

$$
\rho(x, y)=d_{\alpha}\left(\pi_{\alpha}(x), \pi_{\alpha}(y)\right) .
$$

Note that $\rho$ is not a metric or even a quasi-metric. The triangle inequality is violated by any triple such that $x \neq z$ but $y=\pi_{\alpha}(x)=\pi_{\alpha}(z)$.

For $\varepsilon>0$ a subset $A$ of $T$ is an $\varepsilon$-antichain of $T$ if $\rho(x, y)>\varepsilon$ for all distinct $x$ and $y$ in $A$. We say that $T$ is $\varepsilon$-special if there are $\varepsilon$-antichains $A_{n}$, for $n \in \mathbb{N}$, such that $X_{\alpha} \cap \bigcup_{n} A_{n}$ is dense in $X_{\alpha}$, for all $\alpha<\omega_{1}$.

For $\varepsilon>0$ a subset $A$ of $T$ is an $\varepsilon$-branch if $A=\left\{x_{\alpha}: \alpha<\omega_{1}\right\}, \operatorname{Lev}\left(x_{\alpha}\right)=\alpha$ for all $\alpha$, and $\rho\left(x_{\alpha}, x_{\beta}\right) \leq \varepsilon$ for all $\alpha, \beta$. A subtree of $T$ is a subset $S \subseteq T$ that is closed under projection maps and intersects every level $X_{\alpha}$.

Lemma 1.1. The following are equivalent for every metric $\omega_{1}$-tree $T$ and $\varepsilon>0$.

(1) $T$ has an $\varepsilon$-branch,

(2) There is $B \subseteq T$ that intersects cofinally many levels such that $\rho(x, y) \leq \varepsilon$ for all $x, y$ in $B$,

(3) $T$ has a subtree of diameter $\leq \varepsilon$. 
Proof. For $B \subseteq T$ let its downwards closure $S(B)$ be the subset of $T$ such that its intersection with $X_{\alpha}$ is the metric closure of $\left\{\pi_{\alpha}(x): x \in B, \alpha \leq \operatorname{Lev}(x)\right\}$. Since each $\pi_{\alpha}$ us $\rho$-nonincreasing, the ' $\rho$-diameter' of $S(B)$ is equal to the ' $\rho$-diameter' of $B$. This shows that (2) implies (3), and the other implications do not require a proof.

Lemma 1.2. Assume $T$ is a metric $\omega_{1}$-tree such that each of its subtrees has an $\varepsilon$-branch for every $\varepsilon>0$. Then $T$ has a branch.

Proof. Choose $B_{n}$, for $n \in \mathbb{N}$, so that $B_{n}$ is a $1 / n$-branch and $B_{n+1} \subseteq S\left(B_{n}\right)$. Then for every $\alpha$ we have that $B_{n} \cap X_{\alpha}$, for $n \in \mathbb{N}$, is a decreasing sequence of subsets of $X_{\alpha}$ with diameters converging to 0 . If $x_{\alpha}$ is the unique point in $\bigcap_{n}\left(B_{n} \cap X_{\alpha}\right)$ then the fact that the projections are commuting contractions easily implies that $x_{\alpha}$, for $\alpha<\omega_{1}$, is a branch of $T$.

There is a Polish Aronszajn tree with an $\varepsilon$-branch for all $\varepsilon>0$ but no branches. To see this, fix any special Aronszajn tree $T$. Let $X_{\alpha}$ be the disjoint union of countably many copies of the $\alpha$-th level of $T$ and define $d_{\alpha}$ so that the the $n$-th copy has diameter $1 / n$ and the distance between two distinct copies is 1 . With the natural projection maps, the $n$-th copy of $T$ includes a $1 / n$-branch but $T$ has no branches.

In the following lemma and elsewhere no attempt was made to find optimal numerical estimates.

Lemma 1.3. If $T$ is an $\varepsilon$-special metric $\omega_{1}$-tree then it has no $\varepsilon / 2$-branches.

Proof. Let $A_{n}$, for $n \in \mathbb{N}$, be $\varepsilon$-antichains with dense union in each level. Assume $x_{\alpha}$, for $\alpha<\omega_{1}$, is an $\varepsilon$-branch. Let $n$ be such that $d_{\alpha}\left(x_{\alpha}, z_{\alpha}\right)<\varepsilon / 4$ for some $z_{\alpha} \in A_{n} \cap X_{\alpha}$ for uncountably many $\alpha$. Since projections are contractions, for such $\alpha<\beta$ we have $\rho\left(z_{\alpha}, z_{\beta}\right)<\varepsilon$, a contradiction.

The proof of the following lemma is a straightforward modification of the wellknown analogous fact for $\omega_{1}$-trees.

Lemma 1.4 (MA). Assume $T$ is a Polish $\omega_{1}$-tree with no $\varepsilon$-branches. Then $T$ is $\varepsilon / 2$-special.

Proof. For each $\alpha$ fix a countable dense subset $Z_{\alpha}$ of $X_{\alpha}$. Let $\mathbb{P}_{0}$ be the poset of finite $\varepsilon / 2$-antichains included in $\bigcup_{\alpha} Z_{\alpha}$ ordered with $\mathbf{p} \geq \mathbf{q}$ if $\mathbf{p} \subseteq \mathbf{q}$.

We shall prove $\mathbb{P}_{0}$ is ccc. Fix $\mathbf{p}_{\alpha}, \alpha<\omega_{1}$ in $\mathbb{P}_{0}$. Since each $Z_{\alpha}$ is countable, by a $\Delta$ system argument we can find $\bar{\alpha}$, an uncountable $J \subseteq \omega_{1}$, and (writing $Z=\bigcup_{\beta \leq \bar{\alpha}} Z_{\beta}$ ) $\overline{\mathbf{p}} \subseteq Z$ and $\overline{\mathbf{q}} \subseteq Z$ so that the following hold for all $\alpha \in J$. First, $\mathbf{p}_{\alpha}=\overline{\mathbf{p}} \cup \mathbf{q}_{\alpha}$. Second, $\pi_{\bar{\alpha}}$ maps $\mathbf{q}_{\alpha}$ injectively onto $\overline{\mathbf{q}}$. Third, $\gamma(\alpha)=\min \left\{\operatorname{Lev}(x): x \in \mathbf{q}_{\alpha}\right\}$ converges to $\omega_{1}$.

It suffices to find $\alpha<\beta$ in $J$ such that $\mathbf{q}_{\alpha} \cup \mathbf{q}_{\beta}$ is an $\varepsilon / 2$-antichain. Let $n=|\overline{\mathbf{q}}|$ and fix an enumeration $\mathbf{q}_{\alpha}=\left\{z_{\alpha}(i): i<n\right\}$ for all $\alpha \in J$. Let $\mathcal{U}$ be a uniform ultrafilter on $J$. Assuming $\alpha$ and $\beta$ as above cannot be found, there are $i<j<n$ such that the set $J_{1}=\left\{\alpha \in J:\left\{\beta: \rho\left(z_{\alpha}(i), z_{\beta}(j)\right)<\varepsilon / 2\right\} \in \mathcal{U}\right\}$ belongs to $\mathcal{U}$. But then $\rho\left(z_{\alpha}(i), z_{\gamma}(i)\right)<\varepsilon$ for all $\alpha<\gamma$ in $J_{1}$, and therefore $\left\{z_{\alpha}(i): \alpha \in J_{1}\right\}$ defines an $\varepsilon$-branch of $T$.

This proof that $\mathbb{P}_{0}$ is ccc shows that it is powefully ccc, i.e., the finitely supported product $\mathbb{P}_{0}^{<\omega}$ of countably many copies of $\mathbb{P}$ is ccc. Apply MA to the ccc poset $\mathbb{P}=\mathbb{P}_{0}^{<\omega}$ and $\aleph_{1}$ many dense sets assuring that $\mathbb{P}$ ads countably many $\varepsilon$-antichains $A_{n}$ whose union is equal to $\bigcup_{\alpha} Z_{\alpha}$. 
1.1. Coherent families of Polish spaces. The material of this subsection plays a role only in the proof of Theorem 2 and the reader may safely skip it in the first reading.

A system $\mathbb{F}=\left(X_{\lambda}, d_{\lambda}, \pi_{\lambda^{\prime} \lambda}: \lambda<\lambda^{\prime}\right.$ in $\left.\Lambda\right)$ is a coherent family of Polish spaces if

(1) $\Lambda$ is upwards $\sigma$-directed set and a lower semi-lattice,

(2) $X_{\lambda}$ is a Polish space with compatible metric $d_{\lambda}$,

(3) $\pi_{\lambda^{\prime} \lambda}: X_{\lambda^{\prime}} \rightarrow X_{\lambda}$ is a contractive surjection,

(4) projections $\pi_{\lambda^{\prime} \lambda}$ are commuting and $\pi_{\lambda \lambda}=\mathrm{id}_{X_{\lambda}}$ for all $\lambda$.

The family is trivial if $\lim _{\lambda} X_{\lambda} \neq \emptyset$. Hence if $\Lambda=\omega_{1}$ with its natural ordering then $\mathbb{F}$ is a Polish $\omega_{1}$-tree.

Spaces $X_{\lambda}$ are assumed to be disjoint and we shall identify $\mathbb{F}$ with the union $\bigcup_{\lambda} X_{\lambda}$ of its levels when convenient and when the choice of projections is clear from the context. On $\mathbb{F}$ we have a map Lev: $\mathbb{F} \rightarrow \Lambda$ defined by $\operatorname{Lev}(x)=\lambda$ if and only if $x \in X_{\lambda}$. It will be convenient to write $\pi_{\lambda}$ for the map $\bigcup_{\lambda^{\prime}>\lambda} \pi_{\lambda^{\prime} \lambda}$.

Define a map $\rho$ on $\mathbb{F}^{2}$ as follows. For $x, y$ in $\mathbb{F}$ let $\lambda=\operatorname{Lev}(x) \wedge \operatorname{Lev}(y)$ and let

$$
\rho(x, y)=d_{\lambda}\left(\pi_{\lambda}(x), \pi_{\lambda}(y)\right) .
$$

For $\varepsilon>0$ a subset $A$ of $T$ is an $\varepsilon$-antichain of $\mathbb{T}$ if $\rho(x, y)>\varepsilon$ for all distinct $x$ and $y$ in $A$. A set $\left\{x_{\lambda}: \lambda \in \Lambda\right\}$ is an $\varepsilon$-branch of $\mathbb{F}$ if $x_{\lambda} \in X_{\lambda}$ for all $\lambda$ and $\rho\left(x_{\lambda}, x_{\lambda^{\prime}}\right) \leq \varepsilon$ for all $\lambda$ and $\lambda^{\prime}$.

If $Y_{\lambda} \subseteq X_{\lambda}$ is a nonempty Polish subspace for all $\lambda$ and the family $Y_{\lambda}$, for $\lambda \in \Lambda$, is closed under the projection maps then (with $d_{\lambda}^{\prime}$ denoting the restriction of $d_{\lambda}$ to $\left.Y_{\lambda}\right)$ we say that $\mathbb{F}^{\prime}=\left(Y_{\lambda}, d_{\lambda}^{\prime}, \pi_{\lambda^{\prime} \lambda}\right.$, for $\lambda<\lambda^{\prime}$ in $\left.\Lambda\right)$ is a cofinal subfamily of $\mathbb{F}$.

Proof of the following is analogous to the proof of Lemma 1.2.

Lemma 1.5. Assume $\mathbb{F}$ is a coherent family of Polish spaces such that each of its cofinal subfamilies has an $\varepsilon$-branch for every $\varepsilon>0$. Then $\mathbb{F}$ is trivial.

Assume $\mathbb{F}$ is a coherent family of Polish spaces. If $f: \omega_{1} \rightarrow \mathbb{F}$ is a strictly increasing map then we say the Polish $\omega_{1}$-tree $\left(X_{f(\alpha)}, d_{f(\alpha)}, \pi_{f(\beta) f(\alpha)}, \alpha \leq \beta<\omega_{1}\right)$ is a Polish subtree of $\mathbb{F}$.

Lemma 1.6 (PFA). Assume $\mathbb{F}=\left(X_{\lambda}, d_{\lambda}, \pi_{\lambda^{\prime} \lambda}: \lambda<\lambda^{\prime}\right.$ in $\left.\Lambda\right)$ is a coherent family of Polish spaces with no $\varepsilon$-branches. Then $\mathbb{F}$ has an $\varepsilon / 6$-special Polish subtree.

Proof. Let $\mathbb{P}$ denote the $\sigma$-closed collapse of $|\Lambda|$ to $\aleph_{1}$. Then $\mathbb{P}$ forces that there is a strictly increasing, cofinal map $f: \omega_{1} \rightarrow \Lambda$. We first prove that $\mathbb{P}$ forces the Polish $\omega_{1}$-tree $T_{f}=\left(X_{f(\alpha)}, d_{f(\alpha)}, \pi_{f(\beta) f(\alpha)}, \alpha \leq \beta<\omega_{1}\right)$ has no $\varepsilon / 3$-branches.

Assume otherwise and let $\dot{B}$ be a name for an $\varepsilon / 3$-branch of $T_{f}$. Let $\theta=\left(2^{|\Lambda|}\right)^{+}$ and let $M$ be a countable elementary submodel of $H_{\theta}$ containing $\mathbb{F}, \mathbb{P}$, and a name $\dot{f}$ for $f$. Let $\mathcal{D}_{n}$, for $n \in \mathbb{N}$, enumerate all dense open subsets of $\mathbb{P}$ that belong to $M$. Pick conditions $\mathbf{p}_{s}, x_{s}$ and $y_{s}$ for $s \in 2^{<\mathbb{N}}$, satisfying the following for all $s$.

(1) $\mathbf{p}_{s} \geq \mathbf{p}_{t}$ if $t$ extends $s$,

(2) $\mathbf{p}_{s} \in M \cap \mathcal{D}_{n}$, where $n=|s|$,

(3) $\mathbf{p}_{s} \Vdash \check{x}_{s} \in \dot{B}$,

(4) $x_{s} \in M$, and

(5) $\rho\left(x_{s 0}, x_{s 1}\right) \geq \varepsilon$. 
These objects are chosen by recursion. If $\mathbf{p}_{s}$ has been chosen, then the set $\{x \in \mathbb{F}$ : $\left.\left(\exists \mathbf{q} \leq \mathbf{p}_{s}\right) \mathbf{q} \Vdash x \in \dot{B}\right\}$ is not an $\varepsilon$-branch and therefore we can choose $x_{s 0}$ and $x_{s 1}$ in this set such that $\rho\left(x_{s 0}, x_{s 1}\right) \geq \varepsilon$. Let $\mathbf{p}_{s 0}$ and $\mathbf{p}_{s 1}$ be (necessarily incompatible) extensions of $\mathbf{p}_{s}$ forcing that $x_{s 0}$ and $x_{s 1}$, respectively, belong to $\dot{B}$. Since all the relevant parameters are in $M, \mathbf{p}_{s 0}, \mathbf{p}_{s 1}, x_{s 0}$ and $x_{s 1}$ can also be chosen to belong to $M$.

Since $\Lambda$ is $\sigma$-directed, let $\lambda(M) \in \Lambda$ be an upper bound for $M \cap \Lambda$. For each $g \in 2^{\mathbb{N}}$ let $\mathbf{p}_{g}$ be $(M, \mathbb{P})$-generic condition extending all $\mathbf{p}_{g \mid n}$ and deciding $x_{g} \in X_{\lambda(M)}$ in $\dot{B}$. For $g \neq g^{\prime}$ let $s$ be the longest common initial segment of $g$ and $g^{\prime}$. We may assume $g$ extends $s 0$ and $g^{\prime}$ extends $s 1$. Let $\alpha=\min \left(\operatorname{Lev}\left(x_{s 0}\right), \operatorname{Lev}\left(x_{s 1}\right)\right)$ and let $y_{0}, y_{1}, x_{0}, x_{1}$ be the projections of $x_{g}, x_{g^{\prime}}, x_{s 0}$ and $x_{s 1}$, respectively, to $X_{\alpha}$. Then

$$
d_{\alpha}\left(y_{0}, y_{1}\right) \geq d_{\alpha}\left(x_{0}, x_{1}\right)-d_{\alpha}\left(y_{0}, x_{0}\right)-d_{\alpha}\left(y_{1}, x_{1}\right) \geq \varepsilon / 3,
$$

and therefore $d_{\lambda(M)}\left(x_{g}, x_{g^{\prime}}\right) \geq \varepsilon / 3$. This contradicts the assumed separability of $X_{\lambda(M)}$.

Since $\mathbb{P}$ forces that $\mathbb{F}$ has no $\varepsilon / 3$-branches, by Lemma 1.4 we have a $\mathbb{P}$-name for a ccc poset that $\varepsilon / 6$-specializes $T_{f}$. By applying PFA to the iteration and an appropriate collection of dense sets we obtain the desired conclusion.

Coherent families of discrete Polish spaces and their uniformization using PFA have been used in different contexts. See e.g., [12] and [8].

\section{Inner automorphisms}

In this short section we state and prove some well-known results about inner automorphisms of $\mathrm{C}^{*}$-algebras. Recall that for a partial isometry $v$ in algebra $A$ by $\operatorname{Ad} v$ we denote the conjugation map $\operatorname{Ad} v(a)=v a v^{*}$.

Lemma 2.1. Assume that unitaries $v$ and $w$ in a $C^{*}$-algebra $A$ are such that $\operatorname{Ad} v$ and $\operatorname{Ad} w$ agree on $A$. Then $v w^{*} \in \mathcal{Z}(A)$.

Proof. We have $v a v^{*}=w a w^{*}$ and therefore $w^{*} v a=a w^{*} v$ for all $a \in A$.

In the following $\dot{a}$ denotes the image of $a \in \mathcal{B}(H)$ in the Calkin algebra under the quotient map, not a forcing name.

Lemma 2.2. If $v$ and $w$ in $\mathcal{B}(H)$ are such that $\dot{v}$ and $\dot{w}$ are unitaries in $\mathcal{C}(H)$ and $(\operatorname{Ad} v) a-(\operatorname{Ad} w) a$ is compact for all $a \in \mathcal{B}(H)$, then there is $z \in \mathbb{T}$ such that $v-z w$ is compact.

Proof. We first check (a well-known fact) that $\mathcal{Z}(\mathcal{C}(H))=\mathbb{C}$. Since it is a $\mathrm{C}^{*}$-algebra, it suffices to see that the only self-adjoint elements of $\mathcal{Z}(\mathcal{C}(H))$ are scalar multiples of the identity. Assume $\dot{a}$ is self-adjoint and its essential spectrum is not a singleton, say it contains some $\lambda_{1}<\lambda_{2}$. Fix $\varepsilon<\left|\lambda_{1}-\lambda_{2}\right| / 3$. In $\mathcal{B}(H)$ fix infinite-dimensional projections $p$ and $q$ such that $\left\|p a p-\lambda_{1} p\right\|<\varepsilon$ and $\left\|q a q-\lambda_{2} q\right\|<\varepsilon$. A noncompact partial isometry $v$ such that $v v^{*} \leq p$ and $v^{*} v \leq q$ clearly does not commute with $a$ modulo the compacts.

By Lemma 2.1 applied to $\dot{v}$ and $\dot{w}$ and the above there is a scalar $z$ such that $z \dot{v}=\dot{w}$, as required. 
Lemma 2.3. Assume $H$ is an infinite-dimensional Hilbert space and $\Phi$ and $\Psi$ are automorphisms of $\mathcal{C}(H)$ that agree on the corner $\dot{p} \mathcal{C}(H) \dot{p}$ for every projection $p \in$ $\mathcal{B}(H)$ with separable range. Then $\Phi=\Psi$.

Proof. We may assume $H$ is nonseparable. Assume the contrary and let $a \in \mathcal{B}(H)$ be such that $\dot{b}=\Phi(\dot{a})-\Psi(\dot{a}) \neq 0$. Let $r$ be a projection with separable range such that $r b r$ is not compact and let $p$ be such that $\Phi(\dot{p})=\dot{r}$. By our assumption, $\Psi(\dot{p})=\dot{r}$. Also $\dot{r} \Psi(\dot{a}) \dot{r}=\Psi(\dot{p} \dot{a} \dot{p})=\Phi(\dot{p} \dot{a} \dot{p})=\dot{r} \Phi(\dot{a}) \dot{r}$, contradicting the choice of $a$.

\section{Part I of the proof of Theorem 1: Trees $T$ and $T[a]$}

Let $H$ denote $\ell_{2}\left(\aleph_{1}\right)$. Throughout this section we assume $\Phi$ is an automorphism of $\mathcal{C}(H)$ and $\Phi_{*}: \mathcal{B}(H) \rightarrow \mathcal{B}(H)$ is its representation, i.e., any map such that the diagram

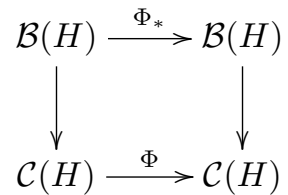

commutes. Since every projection in $\mathcal{C}(H)$ lifts to a projection in $\mathcal{B}(H)([14$, Lemma $3.2]$ ) we may assume $\Phi_{*}$ maps projections to projections.

Lemma 3.1. If $p$ is a projection in $\mathcal{B}(H)$ with separable range, then $\Phi_{*}(p)$ is a projection with separable range and $\Phi(\dot{p} \mathcal{C}(H) \dot{p})=\Phi(\dot{p}) \mathcal{C}(H) \Phi(\dot{p})$.

Proof. Since a nonzero projection in $\mathcal{C}(H)$ generates the minimal nontrivial ideal of $\mathcal{C}(H)$ if and only if it is of the form $\dot{q}$ for some $q$ with a separable range, the first claim follows. For the second part note that $A=\dot{p} \mathcal{C}(H) \dot{p}$ is a hereditary subalgebra (i.e., if $0 \leq a \leq b$ for $a \in \mathcal{C}(H)$ and $b \in A$, then $a \in A$ ) and therefore $\Phi$ maps it to a hereditary subalgebra.

3.1. Localization. A straightfoward recursive construction produces an increasing family of projections with separable range $p_{\alpha}, \alpha<\omega_{1}$ in $\mathcal{B}(H)$ such that

(1) $\bigvee_{\alpha<\omega_{1}} p_{\alpha}=1$ and for a limit $\delta$ we have $p_{\delta}=\bigvee_{\alpha<\delta} p_{\alpha}$,

(2) $p_{0}$ and each $p_{\alpha+1}-p_{\alpha}$ are noncompact,

(3) for some projection $r_{\alpha}$ such that $\dot{r}_{\alpha}=\Phi\left(\dot{p}_{\alpha}\right)$ we have $p_{\alpha} \leq r_{\alpha+1}$ and $r_{\alpha} \leq$ $p_{\alpha+1}$.

For convenience we write $p_{-1}=0$. For each $\alpha$ fix a basis of the range $p_{\alpha+1}-p_{\alpha}$ and enumerate it as $e_{\beta}$, for $\alpha \cdot \omega \leq \beta<(\alpha+1) \cdot \omega$. We therefore have a basis $\left(e_{\alpha}\right)_{\alpha<\omega_{1}}$ for $H$ such that

(4) $p_{\alpha}$ is the closed linear span of $\left\{e_{\beta}: \beta<\alpha \cdot \omega\right\}$.

For every $\alpha<\omega_{1}$ Lemma 3.1 implies that the restriction of $\Phi$ to $\dot{p}_{\alpha} \mathcal{C}(H) \dot{p}_{\alpha}$ is an isomorphism between Calkin algebras associated with separable Hilbert spaces, $p_{\alpha}[H]$ and $r_{\alpha}[H]$. Therefore by Theorem 3 we can fix a partial isometry $v_{\alpha}$ such that

(5) $v_{\alpha} v_{\alpha}^{*} \leq r_{\alpha}, v_{\alpha}^{*} v_{\alpha} \leq p_{\alpha}$, and $\operatorname{Ad} v_{\alpha}$ is a representation of $\Phi$ on $\dot{p}_{\alpha} \mathcal{C}(H) \dot{p}_{\alpha}$.

For each $\alpha>1$ by Lemma 2.2 we can find $z_{\alpha} \in \mathbb{T}$ such that $v_{0}-z_{\alpha} v_{\alpha} p_{0}$ is compact. Replace $v_{\alpha}$ with $z_{\alpha} v_{\alpha}$ and note that $\operatorname{Ad} v_{\alpha}$ still satisfies (5). Let us prove that in addition (with $a={ }^{\mathcal{K}} b$ standing for ' $a-b$ is compact') 
(6) $v_{\alpha}={ }^{\mathcal{K}} v_{\beta} p_{\alpha}$ whenever $\alpha<\beta$.

By Lemma 2.2, there is $z \in \mathbb{T}$ such that $v_{\alpha}-z v_{\beta} p_{\alpha}$ is compact. Since $p_{0}$ is noncompact and since $v_{\alpha} p_{0}={ }^{\mathcal{K}} v_{0}={ }^{\mathcal{K}} v_{\beta} p_{0}$, we must have $z=1$.

For $a \in \mathcal{B}(H)$ define the support of $a$ as

$$
\operatorname{supp}(a)=\left\{\alpha<\omega_{1}:\left\|a e_{\alpha}\right\|>0 \text { or }\left\|a^{*} e_{\alpha}\right\|>0\right\} .
$$

All compact operators are countably supported and the set of finitely supported operators is a dense subset of $\mathcal{K}(H)$. An easy analogue of the $\Delta$-system lemma (e.g., [7, Theorem II.1.5]) is worth stating explicitly (here $H=\ell^{2}\left(\aleph_{1}\right)$ and $p_{\alpha}$ are as in (4)).

Lemma 3.2. Assume $a_{\alpha}, \alpha<\omega_{1}$, belong to $\mathcal{K}(H)$. Then for every $\varepsilon>0$ there is a stationary $X \subseteq \omega_{1}$, a finitely supported projection $r$, and an operator a such that $r a r=a$ and

(a) $\left\|p_{\alpha}\left(r a_{\alpha} r-a_{\alpha}\right) p_{\alpha}\right\|<\varepsilon$ for all $\alpha \in X$,

(b) $\left\|p_{\alpha}\left(a-a_{\alpha}\right) p_{\alpha}\right\|<\varepsilon$ for all $\alpha \in X$, and

(c) $\left\|p_{\alpha} a_{\alpha} p_{\alpha}-p_{\beta} a_{\beta} p_{\beta}\right\|<2 \varepsilon$ for all $\alpha<\beta$ in $X$.

Proof. For $a_{\alpha}$ find a finitely supported $b_{\alpha}$ with complex rational coefficients with support in $p_{\alpha}$ such that $\left\|p_{\alpha}\left(a_{\alpha}-b_{\alpha}\right) p_{\alpha}\right\|<\varepsilon / 2$. By the Pressing Down Lemma ([7, Theorem II.6.15]) we can find a stationary set $X_{0}$ such that all $b_{\alpha}$ with $\alpha \in X_{0}$ have the same support, $S$. Let $r$ be the projection to $\operatorname{span}\left\{e_{i}: i \in S\right\}$. By a counting argument we can refine $X_{0}$ further and find $a$. The third inequality is an immediate consequence of the second.

3.2. The tree $T$. For $\alpha<\omega_{1}$ let (with $r_{\alpha}$ and $p_{\alpha}$ as in (3) of $\S 3.1$ )

$$
X_{\alpha}=\left\{r_{\alpha+1} w p_{\alpha}: w \in \mathcal{B}(H), w={ }^{\mathcal{K}} v_{\alpha}\right\} .
$$

Note the 'extra room' provided by defining $X_{\alpha}$ in this way instead of the apparently more natural $\left\{r_{\alpha} w p_{\alpha}: w \in \mathcal{B}(H), w={ }^{\mathcal{K}} v_{\alpha}\right\}$. Let us prove a few properties of $X_{\alpha}$.

(7) $X_{\alpha}$ is a norm-separable complete metric space.

(8) If $\alpha<\beta$ then the map $\pi_{\beta \alpha}: X_{\beta} \rightarrow X_{\alpha}$ defined by

$$
\pi_{\beta \alpha}(w)=r_{\alpha+1} w p_{\alpha}
$$

is a surjection and a contraction.

Only the latter property requires a proof. It is clear that the range of $\pi_{\beta \alpha}$ is included in $X_{\alpha}$ and that the map is contraction. For $u \in X_{\alpha}$ let $w=v_{\beta}+u-r_{\alpha+1} v_{\beta} p_{\alpha}$. Then $w-v_{\beta}$ is compact since $u \in X_{\alpha}$ and clearly $r_{\alpha+1} w p_{\alpha}=r_{\alpha+1} u p_{\alpha}=u$.

Consider the Polish $\omega_{1}$-tree $T$ with levels $X_{\alpha}$ and connecting maps $\pi_{\alpha \beta}$.

Lemma 3.3. The following are equivalent.

(9) $\Phi$ is inner.

(10) There is a $v \in \mathcal{B}(H)$ such that $\dot{v}$ is a unitary in $\mathcal{C}(H)$ and for all $\alpha<\omega_{1}$ we have $r_{\alpha+1} v p_{\alpha} \in X_{\alpha}$.

(11) Thas a branch.

Proof. Clearly (10) and (11) are equivalent, hence it suffices to prove (9) implies (10) and that (10) implies (11). Assume $\Phi$ is inner and $v$ implements it. Then by Lemma 2.2 for every $\alpha<\omega_{1}$ there is $z_{\alpha} \in \mathbb{T}$ such that $z_{\alpha} v p_{\alpha}-v_{\alpha}$ is compact. Since 
$v_{\alpha} p_{0}-v_{0}$ is compact for each $\alpha$ and $p_{0}$ is noncompact, we have $z_{\alpha}=z_{0}$ for all $\alpha$. Therefore $z_{0} v$ defines a branch of $T$.

Now assume (11) and fix a $v$ that defines a branch of $T$. Then the automorphism of $\mathcal{C}(H)$ with representation $\operatorname{Ad} v$ agrees with $\Phi$ on the ideal of all operators with separable range. By Lemma 2.3, this automorphism agrees with $\Phi$ on all of $\mathcal{C}(H)$, hence (9) follows.

A minor modification of the proof that (10) implies (11) above gives an another equivalent reformulation of $\Phi$ being inner. Although we shall not need it, it deserves mention:

(12) Every subtree of $T$ has a branch.

We proceed with the analysis of $T$ and the corresponding 'local trees' $T[a]$.

For $b \in \mathcal{B}(H)$ and $\alpha<\omega_{1}$ let

$$
Z[b]_{\alpha}=\left\{p_{\alpha} w b w^{*} p_{\alpha}: w \in X_{\alpha+1}\right\} .
$$

Then for every $c \in Z[b]_{\alpha}$ we have $p_{\alpha} \Phi_{*}(b) p_{\alpha}={ }^{\mathcal{K}} c$ because

$$
\begin{aligned}
p_{\alpha} w b w^{*} p_{\alpha} & ={ }^{\mathcal{K}} p_{\alpha} v_{\alpha+1} p_{\alpha+1} b p_{\alpha+1} v_{\alpha+1}^{*} p_{\alpha} \\
& ={ }^{\mathcal{K}} p_{\alpha} \Phi_{*}\left(p_{\alpha+1} b p_{\alpha+1}\right) p_{\alpha} \\
& ={ }^{\mathcal{K}} p_{\alpha} r_{\alpha+1} \Phi_{*}(b) r_{\alpha+1} p_{\alpha} \\
& ={ }^{\mathcal{K}} p_{\alpha} \Phi_{*}(b) p_{\alpha} .
\end{aligned}
$$

Also, for $\alpha<\beta$ the map $\varpi_{\beta \alpha}^{b}$ (denoted $\varpi_{\beta \alpha}$ when $b$ is clear from the context) from $Z[b]_{\beta}$ to $Z[b]_{\alpha}$ defined by

$$
\varpi_{\beta \alpha}(c)=p_{\alpha} c p_{\alpha}
$$

is clearly a contractive surjection.

For $a \in \mathcal{B}(H)$ let $T[a]$ denote the Polish $\omega_{1}$-tree with levels $Z[a]_{\alpha}$ and commuting projections $\varpi_{\beta \alpha}$. By 'subtree' we always mean a downwards closed subtree of height $\omega_{1}$.

Lemma 3.4. For every $a \in \mathcal{B}(H)$ every subtree $S$ of $T[a]$ has a branch.

Proof. Let $b=\Phi_{*}(a)$. For every $\alpha<\omega_{1}$ fix $w_{\alpha} \in X_{\alpha+1}$ such that

$$
b_{\alpha}=p_{\alpha} w_{\alpha} a w_{\alpha}^{*} p_{\alpha}
$$

belongs to $S \cap Z[a]_{\alpha}$. Let $u_{\alpha}=p_{\alpha} w_{\alpha}$.

Fix $\varepsilon>0$. Recall that the fixed basis $e_{\alpha}$, for $\alpha<\omega_{1}$, of $H$ spans all $p_{\alpha}$ (see (4)). Apply ' $\Delta$-system' Lemma 3.2 to operators $p_{\alpha}\left(b-b_{\alpha}\right) p_{\alpha}$ to find uncountable $J \subseteq \omega_{1}$ and finitely supported $c$ and $c_{\alpha}, \alpha \in J$, with disjoint supports, so that

$$
\left\|\left(b-b_{\alpha}\right)-\left(c+c_{\alpha}\right)\right\|<\varepsilon \text { and }\left\|p_{\alpha}\left(b-b_{\alpha}\right) p_{\alpha}-c\right\|<\varepsilon .
$$

By going to a further subset of $J$ we may assume that for $\alpha<\beta$ in $J$ the support of $c_{\alpha}$ is included in $\beta \cdot \omega$ (or more naturally stated, that $p_{\beta} c_{\alpha} p_{\beta}=c_{\alpha}$ ). For each $\alpha \in J$ let $\alpha^{+}$be the minimal element of $J$ above $\alpha$ and let $b_{\alpha}^{\prime}=p_{\alpha}\left(b_{\alpha^{+}}\right) p_{\alpha}$. For $\alpha$ in $J$ we have $\left\|b_{\alpha}^{\prime}-\left(p_{\alpha} b p_{\alpha}-c\right)\right\|<\varepsilon$, and therefore $\left\|b_{\alpha}^{\prime}-p_{\alpha} b_{\beta}^{\prime} p_{\alpha}\right\|<2 \varepsilon$ for $\alpha<\beta$ in $J$. Hence $b_{\alpha}^{\prime}$, for $\alpha \in J$, defines a $2 \varepsilon$-branch in $T[a]$. Since $S$ has a $2 \varepsilon$-branch for an arbitrarily small $\varepsilon$ it has a branch by Lemma 1.2 . 


\section{Proof of Theorem 1, part II: A generic operator}

In this section we apply Martin's Axiom. First, we add a generic operator $\tau$ to $\mathcal{B}(H)$ by a poset with finite conditions which forces that $T[\tau]$ has a branch. Second, we use the properties of $\tau$ to $\operatorname{argue}$ that $T$ has a branch.

4.1. Adding $\aleph_{1}$ Cohen reals. For a Hilbert space $K$ with a fixed basis $e_{j}, j \in J$, let $\mathbb{P}(K)$ be the forcing defined as follows. A condition in $\mathbb{P}(K)$ is a pair $(F, M)$ where $F$ is a finite subset of $J$ and $M$ is an $F \times F$ matrix with entries in the complex rationals, $\mathbb{Q}+i \mathbb{Q}$, such that the operator norm of $M$ satisfies $\|M\|<1$. We order $\mathbb{P}(K)$ by extension, setting $\left(F^{\prime}, M^{\prime}\right) \leq(F, M)$ if $F^{\prime} \supseteq F$ and $M^{\prime} \uparrow F \times F \equiv M$.

Lemma 4.1. Poset $\mathbb{P}(K)$ is ccc if and only if $K$ is separable.

Proof. if $K$ is separable then $\mathbb{P}(K)$ is countable, so we only need to show the other direction. This direction will not be used in our proof, but we nevertheless include it since it shows why Lemma 4.2 below does not use $\mathbb{P}(H)$.

We may assume $0 \in J$. For each $j \in J \backslash\{0\}$ define a condition $\mathbf{a}_{j}=\left(F^{j}, M^{j}\right)$ by $F^{j}=\{0, j\}$ and the $(0, j)$ entry of $M^{j}$ is equal to $1 / \sqrt{2}$, while the other three entries are 0 . Then the norm of any matrix including $M_{j}$ and $M_{k}$ is at least 1 , hence $a_{j}$, for $j \in J$, is an uncountable antichain.

4.2. Adding a generic operator $\tau$. By (2) in $\S 3.1$ the projection

$$
s_{\alpha}=p_{\alpha+1}-p_{\alpha}
$$

has an infinite-dimensional and separable range. Let

$$
\mathcal{D}=\left\{a \in \mathcal{B}(H): a=\sum_{\alpha<\omega_{1}} s_{\alpha} a s_{\alpha}\right\}
$$

where the sum is taken in the strong operator topology. This subalgebra of $\mathcal{B}(H)$ is an analogue of algebras $\mathcal{D}[\vec{E}]$ that played a prominent part in the proof of Theorem 3 in [5]. Although much of the theory of $\mathcal{D}[\vec{E}]$ has analogues in the nonseparable case, we shall not develop this theory since the role of $\mathcal{D}$ in the proof of Theorem 1 is different.

For each $\alpha<\omega_{1}$ let $H_{\alpha}=s_{\alpha} H$, with the basis $\left\{e_{\xi}: \alpha \cdot \omega \leq \xi<(\alpha+1) \cdot \omega\right\}$ and let $\mathbb{P}_{\alpha}$ be $\mathbb{P}\left(H_{\alpha}\right)$. The finitely supported product $\mathbb{P}$ of $\mathbb{P}_{\alpha}$, for $\alpha<\omega_{1}$ is ccc. Actually, being a finitely supported product of countable posets, it is forcing-equivalent to the poset for adding $\aleph_{1}$ Cohen reals.

If $\dot{G} \subseteq \mathbb{P}$ is a generic filter, then it defines a sesquilinear form whose norm is, by genericity, equal to 1 . This in turn defines an operator on $H$ in the unit ball of $\mathcal{B}(H)$ ([9, Lemma 3.2.2]) This operator belongs to the von Neumann algebra $\mathcal{D}$ (see $\S 4.2$ ) and we let $\tau$ denote its $\mathbb{P}$-name.

Lemma 4.2. Poset $\mathbb{P}$ forces that every subtree of $T[\tau]$ has a branch.

Proof. If not, then by Lemma 3.4 we fix a condition $p \in \mathbb{P}$ deciding $\varepsilon>0$ such that some subtree $T^{\prime}[\tau]$ of $T[\tau]$ has no $\varepsilon$-branch and consider $\mathbb{P} * \dot{\mathbb{S}}$ (below $p$ ) where $\dot{\mathbb{S}}$ is a ccc poset for $\varepsilon / 2$-specializing $T^{\prime}[\tau]$. By applying MA we can find $a \in \mathcal{B}(H)$ and an $\varepsilon / 2$-special subtree of $T[a]$. By Lemma 1.3 this subtree has no branches, and this contradicts Lemma 3.4. 
Fix $\varepsilon>0$. By Lemma 4.2, if $S$ is a subtree of $T$ then for $\alpha<\omega_{1}$ we can fix $w_{\alpha}$ and a condition $\mathbf{a}_{\alpha}$ in $\mathbb{P}$ that forces $\operatorname{Ad}\left(p_{\alpha} w_{\alpha}\right) \tau$ belongs to a cofinal $\varepsilon$-branch of $T[\tau]$. Here $w_{\alpha} \in S \cap X_{\alpha+1}$ and $w_{\alpha}$ is in the ground model. Identify $\mathbf{a}_{\alpha}$ with a finitely supported operator in $\mathcal{B}(H)$ and note that it belongs to the algebra $\mathcal{D}$ as defined in $\S 4.2$. Apply Lemma 3.2 to $\left\{\operatorname{Ad}\left(p_{\alpha} w_{\alpha}\right) \mathbf{a}_{\alpha}\right\}$ to find a finitely supported $\mathbf{b}$ such that

(13) $\left\|\mathbf{b}-\operatorname{Ad}\left(p_{\alpha} w_{\alpha}\right) \mathbf{a}_{\alpha}\right\|<\varepsilon$

for all $\alpha$ in a stationary set $J_{0}$. Since the coefficients of $\mathbf{a}_{\alpha}$ are complex rationals, by the $\Delta$-system lemma and a counting argument there are a stationary set $J_{1} \subseteq J_{0}$, a finitely-supported projection $q$, and a such that

(14) $q \mathbf{a} q=\mathbf{a}$ and $p_{\alpha} \mathbf{a}_{\alpha} p_{\alpha}=\mathbf{a}$

for all $\alpha \in J_{1}$. Note that $\mathbf{a}_{\alpha}=\mathbf{a}+\left(I-p_{\alpha}\right) \mathbf{a}_{\alpha}\left(I-p_{\alpha}\right)$ for all $\alpha \in J_{1}$. Find $\bar{\alpha}$ such that $p_{\bar{\alpha}} q=q$. Applying Lemma 3.2 to $\left(w_{\beta}-v_{\bar{\alpha}}\right) p_{\bar{\alpha}}$ find a stationary $J \subseteq J_{1}$ such that

(15) $\left\|\left(w_{\beta}-w_{\gamma}\right) p_{\bar{\alpha}}\right\|<\varepsilon$

for all $\beta<\gamma$ in $J$. Let $q_{\alpha}$ denote the support of $\mathbf{a}_{\alpha}$. For $\beta \in J$ let $u_{\beta}=w_{\beta} p_{\beta}$. Then for $\alpha+1 \leq \beta$ we have $p_{\alpha} u_{\beta}={ }^{\mathcal{K}} p_{\alpha} w_{\beta}$.

Lemma 4.3. The set $\left\{r_{\alpha+2} u_{\beta} p_{\alpha+1}: \alpha+\omega<\beta, \beta \in J\right\}$ is a $5 \varepsilon$-branch of $T$.

Preparations for the proof of Lemma 4.3 take up the remainder of this section, with the main points being Claim 4.6 and Lemma 4.7.

Claim 4.4. If $a \in \mathcal{D}, \alpha<\beta$ are in $J, q_{\alpha} a q_{\alpha}=\mathbf{a}_{\alpha}$, and $q_{\beta} a q_{\beta}=\mathbf{a}_{\beta}$, then

$$
\left\|\operatorname{Ad}\left(p_{\alpha} w_{\alpha}\right) a-\operatorname{Ad}\left(p_{\alpha} w_{\beta}\right) a\right\| \leq \varepsilon .
$$

Proof. Otherwise, there is $\delta>0$ and a finitely supported projection $s \geq q_{\alpha} \vee q_{\beta}$ such that for every $c \in \mathcal{D}$ satisfying scs $=$ sas we have $\left\|\operatorname{Ad}\left(p_{\alpha} w_{\alpha}\right) c-\operatorname{Ad}\left(p_{\alpha} w_{\beta}\right) c\right\|>\varepsilon+\delta$. Making a small change to coefficients of sas one obtains a condition in $\mathbb{P}$ forcing that $\left\|\operatorname{Ad}\left(p_{\alpha} w_{\alpha}\right) \tau-\operatorname{Ad}\left(p_{\alpha} w_{\beta}\right) \tau\right\|>\varepsilon$, a contradiction.

Claim 4.5. Assume $a$ and $b$ are in $\mathcal{D}$, $q a q=q b q=0, p_{\alpha} a p_{\alpha+\omega}=p_{\alpha} b p_{\alpha+\omega}$, and $\alpha+\omega<\beta$ for $\beta \in J$. Then

$$
\left\|\operatorname{Ad}\left(p_{\alpha} w_{\beta}\right)\left(a+\mathbf{a}_{\beta}\right)-\operatorname{Ad}\left(p_{\alpha} w_{\beta}\right)\left(b+\mathbf{a}_{\beta}\right)\right\| \leq 2 \varepsilon .
$$

Proof. Assume otherwise and let

$$
\delta=\left\|\operatorname{Ad}\left(p_{\alpha} w_{\beta}\right)\left(a+\mathbf{a}_{\beta}\right)-\operatorname{Ad}\left(p_{\alpha} w_{\beta}\right)\left(b+\mathbf{a}_{\beta}\right)\right\|-2 \varepsilon .
$$

For $n<\omega$ write $s_{n}=p_{\alpha+\omega}-p_{\alpha+n}$. By continuity fix $n<\omega$ such that for all $c \in s_{n} \mathcal{D}$ $\left(=s_{n} \mathcal{D} s_{n}\right.$ since $s_{n}$ in the commutant of $\left.\mathcal{D}\right)$ with $\|c\| \leq 1$ we have

$$
\left\|\operatorname{Ad}\left(p_{\alpha} w_{\beta}\right)\left(a+\mathbf{a}_{\beta}\right)-\operatorname{Ad}\left(p_{\alpha} w_{\beta}\right)\left(\left(1-s_{n}\right)\left(a+\mathbf{a}_{\beta}\right)+c\right)\right\|<\delta / 2
$$

and

$$
\left\|\operatorname{Ad}\left(p_{\alpha} w_{\beta}\right)\left(b+\mathbf{a}_{\beta}\right)-\operatorname{Ad}\left(p_{\alpha} w_{\beta}\right)\left(\left(1-s_{n}\right)\left(b+\mathbf{a}_{\beta}\right)+c\right)\right\|<\delta / 2 .
$$

Let $c=\mathbf{a}_{\alpha+n}-\mathbf{a}$. Then Claim 4.4 applied to $\left(1-s_{n}\right)\left(a+\mathbf{a}_{\beta}\right)+c$ and to $\left(1-s_{n}\right)(b+$ $\left.\mathbf{a}_{\beta}\right)+c$ implies

$$
\begin{array}{r}
\left\|\operatorname{Ad}\left(p_{\alpha} w_{\beta}\right)\left(\left(1-s_{n}\right)\left(a+\mathbf{a}_{\beta}\right)+c\right)-\operatorname{Ad}\left(p_{\alpha} w_{\alpha+n}\right)\left(\left(1-s_{n}\right)\left(a+\mathbf{a}_{\beta}\right)+c\right)\right\| \leq \varepsilon \\
\left\|\operatorname{Ad}\left(p_{\alpha} w_{\beta}\right)\left(\left(1-s_{n}\right)\left(b+\mathbf{a}_{\beta}\right)+c\right)-\operatorname{Ad}\left(p_{\alpha} w_{\alpha+n}\right)\left(\left(1-s_{n}\right)\left(b+\mathbf{a}_{\beta}\right)+c\right)\right\| \leq \varepsilon
\end{array}
$$

leading to $2 \varepsilon+\delta<2 \varepsilon+\delta$. 
Claim 4.6. For $\alpha+\omega<\beta<\gamma$ such that $\beta$ and $\gamma$ are in $J$ we have

$$
\Delta=\left\|\operatorname{Ad}\left(p_{\alpha} u_{\beta}\right) a-\operatorname{Ad}\left(p_{\alpha} u_{\gamma}\right) a\right\| \leq 5 \varepsilon
$$

for all $a \in \mathcal{D}$ with $\|a\| \leq 1$ and $\left(1-p_{\beta}\right) a=0$.

Proof. Fix $a \in \mathcal{D}$ with $\|a\| \leq 1$. We have that $\mathbf{c}=\mathbf{a}_{\beta}+\left(1-p_{\gamma}\right) \mathbf{a}_{\gamma}$ is a condition in $\mathbb{P}$ with support $q^{\prime}=q_{\beta} \vee q_{\gamma}$ extending both $\mathbf{a}_{\beta}$ and $\mathbf{a}_{\gamma}$. Let

$$
a^{\prime}=a-q^{\prime} a q^{\prime}+\mathbf{c} .
$$

With $\bar{\alpha}$ as in $(15)$ we have $p_{\bar{\alpha}} a=a p_{\bar{\alpha}}$ since $a \in \mathcal{D}$. Therefore

$$
\begin{aligned}
\operatorname{Ad}\left(p_{\alpha} u_{\beta}\right) a-\operatorname{Ad}\left(p_{\alpha} u_{\beta}\right) a^{\prime} & =\operatorname{Ad}\left(p_{\alpha} u_{\beta} p_{\bar{\alpha}}\right)\left(a-a^{\prime}\right)+\operatorname{Ad}\left(p_{\alpha} u_{\beta}\left(p_{\beta}-p_{\bar{\alpha}}\right)\right)\left(a-a^{\prime}\right) \\
& =\operatorname{Ad}\left(p_{\alpha} u_{\beta} p_{\bar{\alpha}}\right)\left(a-a^{\prime}\right) .
\end{aligned}
$$

By this and an analogous computation for $\gamma$ we have

$$
\begin{aligned}
\operatorname{Ad}\left(p_{\alpha} u_{\beta}\right) a-\operatorname{Ad}\left(p_{\alpha} u_{\gamma}\right) a= & \operatorname{Ad}\left(p_{\alpha} u_{\beta} p_{\bar{\alpha}}\right)\left(a-a^{\prime}\right)-\operatorname{Ad}\left(p_{\alpha} u_{\gamma} p_{\bar{\alpha}}\right)\left(a-a^{\prime}\right) \\
& +\operatorname{Ad}\left(p_{\alpha} u_{\beta}\right) a^{\prime}-\operatorname{Ad}\left(p_{\alpha} u_{\gamma}\right) a^{\prime}
\end{aligned}
$$

Using (15) and $p_{\beta} \mathbf{a}_{\beta}=p_{\gamma} \mathbf{a}_{\gamma}=\mathbf{a}$ we conclude that each of the first two summands has norm $\leq \varepsilon$, hence $\Delta$ is within $2 \varepsilon$ of $\left\|\operatorname{Ad}\left(p_{\alpha} u_{\beta}\right) a^{\prime}-\operatorname{Ad}\left(p_{\alpha} u_{\gamma}\right) a^{\prime}\right\|$. Since $a^{\prime} \in \mathcal{D}$ we have $\left(1-p_{\beta}\right) a^{\prime}=\left(1-p_{\beta}\right) \mathbf{a}_{\beta}$ and the following.

$$
\operatorname{Ad}\left(p_{\alpha} u_{\beta}\right) a^{\prime}=\operatorname{Ad}\left(p_{\alpha} w_{\beta}\right) a^{\prime}-\operatorname{Ad}\left(w_{\beta}\left(1-p_{\beta}\right)\right) \mathbf{a}_{\beta} .
$$

By this and an analogous computation for $\gamma$ we have

$$
\begin{aligned}
\operatorname{Ad}\left(p_{\alpha} u_{\beta}\right) a^{\prime}-\operatorname{Ad}\left(p_{\alpha} u_{\gamma}\right) a^{\prime}= & \operatorname{Ad}\left(p_{\alpha} w_{\beta}\right) a^{\prime}-\operatorname{Ad}\left(p_{\alpha} w_{\gamma}\right) a^{\prime} \\
& +\operatorname{Ad}\left(w_{\beta}\left(1-p_{\beta}\right)\right) \mathbf{a}_{\beta}-\operatorname{Ad}\left(w_{\gamma}\left(1-p_{\gamma}\right)\right) \mathbf{a}_{\gamma} .
\end{aligned}
$$

By Claim 4.4 the first difference has norm $\leq \varepsilon$ and by (13) the second difference has norm $\leq 2 \varepsilon$. The conclusion follows.

4.3. Metrics on $X_{\alpha+1}$. We are now within one page worth of definitions and computations from completing the proof. In order to complement Claim 4.6 in the proof of Lemma 4.3, we digress a little bit. For $\alpha<\omega_{1}$ define the following metrics on $X_{\alpha+1}$ (only $d_{4}$ and $d_{2}$ will be needed in our proof).

$$
\begin{aligned}
d_{1, \alpha}(u, w) & =\|u-w\| \\
d_{2, \alpha}(u, w) & =\sup _{a \in \mathcal{D},\|a\|=1}\|\operatorname{Ad} u a-\operatorname{Ad} w a\| \\
d_{3, \alpha}(u, w) & =\sup _{a \in \mathcal{B}(H),\|a\|=1}\|\operatorname{Ad} u a-\operatorname{Ad} w a\| \\
d_{4, \alpha}(u, w) & =\left\|p_{\alpha}(u-w)\right\|
\end{aligned}
$$

We shall drop the subscript $\alpha$ whenever it is clear from the context.

Lemma 4.7. For all $\alpha$, on $X_{\alpha+1}$ we have $d_{4} \leq d_{2} \leq d_{3} \leq 2 d_{1}$.

Proof. The inequality $d_{2} \leq d_{3}$ is trivial, and $d_{3} \leq 2 d_{1}$ follows from the following computation.

$$
\begin{aligned}
\|\operatorname{Ad} u a-\operatorname{Ad} w a\| & \leq\left\|u a u^{*}-u a w^{*}\right\|+\left\|u a w^{*}-w a w^{*}\right\| \\
& \leq\|u a\| \cdot\left\|u^{*}-w^{*}\right\|+\|u-w\| \cdot\|u a\|
\end{aligned}
$$


It remains to prove $d_{4} \leq d_{2}$.

Let $v, w \in X_{\alpha+1}$ be given, and put $d=\left\|p_{\alpha}(v-w)\right\|$. Fix $\delta>0$ and a unit vector $\xi$ such that $\left\|\left(v^{*}-w^{*}\right) p_{\alpha} \xi\right\|>d-\delta$. Clearly we may assume $p_{\alpha} \xi=\xi$. Let $\zeta$ be a unit vector colinear with $v^{*} \xi-w^{*} \xi$ and let $\iota$ be a unit vector orthogonal to $\zeta$ such that $v^{*} \xi$ and $w^{*} \xi$ belong to the linear span of $\zeta$ and $\iota$. Fix scalars $x, y, x^{\prime}, y^{\prime}$ such that

$$
\begin{gathered}
v^{*} \xi=x \zeta+y \iota \\
w^{*} \xi=x^{\prime} \zeta+y^{\prime} \iota
\end{gathered}
$$

Since $v^{*} \xi-w^{*} \xi$ is colinear with $\zeta$, we have $y=y^{\prime}$. Therefore $\left\|v^{*} \xi-w^{*} \xi\right\|=\left|x-x^{\prime}\right|$.

Find representations $\zeta=\sum_{\gamma<\alpha} x_{\gamma} \zeta_{\gamma}$ and $\iota=\sum_{\gamma<\alpha} y_{\gamma} \iota_{\gamma}$ so that $\zeta_{\gamma}$ and $\iota_{\gamma}$ belong to the range of $s_{\gamma}=p_{\gamma+1}-p_{\gamma}$ for all $\gamma$. Since the range of $s_{\gamma}$ is infinite-dimensional and since $v-w$ is compact, we can find a unit vector $\nu_{\gamma}$ in this range orthogonal to both $\zeta_{\gamma}$ and $\iota_{\gamma}$ and such that $\left\|v \nu_{\gamma}\right\|=1$ but $\left\|v \nu_{\gamma}-w \nu_{\gamma}\right\|<\delta / d$. Let

$$
\nu=\sum_{\gamma<\alpha} x_{\gamma} \nu_{\gamma}
$$

Then $\zeta, \iota$, and $\nu$ are mutually orthogonal unit vectors and the rank two operator $a \in \mathcal{B}(H)$ defined by $a(\nu)=\zeta$ and $a(\zeta)=\nu$ has norm equal to one. Moreover, $a \in \mathcal{D}$, since for each $\gamma$ the operator $a s_{\gamma}=s_{\gamma} a$ is just the rank-two operator which transposes the orthogonal unit vectors $\nu_{\gamma}$ and $\zeta_{\gamma}$. Note that $((\operatorname{Ad} v) a) \xi=v a v^{*} \xi=v a(x \zeta+y \iota)=$ $x w \nu$ and $((\operatorname{Ad} w) a) \xi=w a w^{*} \xi=w a\left(x^{\prime} \zeta+y \iota\right)=x^{\prime} w \nu$. Hence,

$$
\|((\operatorname{Ad} v) a-(\operatorname{Ad} w) a) \xi\|=\left\|\left(x-x^{\prime}\right) w \nu\right\|=\left|x-x^{\prime}\right|>d-\delta .
$$

Since $\delta>0$ was arbitrary, we conclude that $d_{2}(v, w) \geq d$.

Proof of Lemma 4.3. In order to show $\left\{r_{\alpha+2} u_{\beta} p_{\alpha+1}: \alpha+\omega<\beta, \beta \in J\right\}$ is a $5 \varepsilon$ branch, it suffices to show that $\left\|p_{\alpha+3}\left(u_{\beta}-u_{\gamma}\right) p_{\alpha+2}\right\| \leq 5 \varepsilon$ whenever $\alpha+\omega<\beta<\gamma$ for $\beta, \gamma$ in $J$. But the inequality $d_{4, \alpha+1} \leq d_{2, \alpha+1}$ from Lemma 4.7 implies

$$
\left\|p_{\alpha+3}\left(u_{\beta}-u_{\gamma}\right) p_{\alpha+2}\right\| \leq \sup _{a \in \mathcal{D}}\left\|\operatorname{Ad}\left(p_{\alpha+3} u_{\beta} p_{\alpha+2}\right) a-\operatorname{Ad}\left(p_{\alpha+3} u_{\gamma} p_{\alpha+2}\right) a\right\|
$$

and the right hand side is $\leq 5 \varepsilon$ by Claim 4.6

Since $\varepsilon$ was arbitrary, Lemma 4.3 and Lemma 1.2 imply that $T$ has a cofinal branch. By Lemma 3.3, $\Phi$ is inner.

\section{The proof of Theorem 2}

The proof of Theorem 2 is reasonably similar to the proof of the analogous result from $[13, \S 4]$. All we need is the analysis of coherent families of Polish spaces from $\S 1.1$ and a fragment of PFA. Fix $\kappa \geq \aleph_{2}$, write $H=\ell^{2}(\kappa)$ and let $\Phi$ be an automorphism of the Calkin algebra $\mathcal{C}(H)$. Fix a basis $\left\{e_{\alpha}: \alpha<\kappa\right\}$ of $H$ and denote the projection to $\overline{\operatorname{span}\left\{e_{\alpha}: \alpha \in \lambda\right\}}$ by $p_{\lambda}$.

Recall that $\mathcal{P}_{\omega_{1}}(\kappa)$ denotes the family of all countable subsets of $\kappa$. This set is $\sigma$ directed under the inclusion and it is a lower semilattice. For every countable subset $\lambda \subseteq \kappa$ fix projection $r_{\lambda}$ with separable range such that $\Phi\left(\dot{p}_{\lambda}\right)=\dot{r}_{\lambda}$. For $\lambda \leq \lambda^{\prime}$ in $\Lambda$ we have $\dot{r}_{\lambda} \leq \dot{r}_{\lambda^{\prime}}$ but not necessarily $r_{\lambda} \leq r_{\lambda^{\prime}}$. By [5] we can fix a partial isometry $v_{\lambda}$ such that $\operatorname{Ad} v_{\lambda}$ implements the restriction of $\Phi$ to $\dot{p}_{\lambda} \mathcal{C}(H) \dot{p}_{\lambda}$. For $\lambda \in \mathcal{P}_{\omega_{1}}(\kappa)$ let

$$
X_{\lambda}=\left\{r_{\lambda} w p_{\lambda}: w \in \mathcal{B}(H), w={ }^{\mathcal{K}} v_{\lambda}\right\} .
$$

Let us prove a few properties of $X_{\lambda}$. 
(16) $X_{\lambda}$ is a norm-separable complete metric space.

(17) If $\lambda \subseteq \lambda^{\prime}$ then the map $\pi_{\lambda^{\prime} \lambda}: X_{\lambda^{\prime}} \rightarrow X_{\lambda}$ defined by

$$
\pi_{\lambda^{\prime} \lambda}(w)=r_{\lambda} w p_{\lambda}
$$

is a contraction.

The proof is analogous to the proof of (8) in $\S 3.2$.

Consider the coherent family of Polish spaces

$$
\mathbb{F}=\left(X_{\lambda}, \pi_{\lambda^{\prime} \lambda}, \pi_{\lambda^{\prime} \lambda}, \text { for } \lambda \in \mathcal{P}_{\omega_{1}}(\kappa)\right) .
$$

The omitted proof of the following uses Lemma 2.3 and is analogous to the proof of Lemma 3.3.

Lemma 5.1. The following are equivalent.

(18) $\Phi$ is inner.

(19) There is $v \in \mathcal{B}(H)$ such that $\dot{v}$ is a unitary in $\mathcal{C}(H)$ and for all $\lambda \in \mathcal{P}_{\omega_{1}}(\kappa)$ we have $r_{\lambda} v p_{\lambda} \in X_{\lambda}$.

(20) The coherent family of Polish spaces $\mathbb{F}$ is trivial.

If $\Phi$ is not inner, then by Lemma 5.1 and Lemma 1.5 there is an $\varepsilon>0$ and a cofinal subfamiy $\mathbb{F}^{\prime}$ of $\mathbb{F}$ with no $\varepsilon$-branches. By PFA and Lemma 1.6, there is a strictly increasing map $f: \omega_{1} \rightarrow \mathbb{F}$ such that the Polish $\omega_{1}$-tree $\left(X_{f(\alpha)}, d_{f(\alpha)}, \pi_{f(\beta) f(\alpha)}, \alpha \leq\right.$ $\left.\beta<\omega_{1}\right)$ is $\varepsilon / 6$-special. Then $Z=\bigcup f\left[\omega_{1}\right]$ is an $\aleph_{1}$-sized subset of $\kappa$. Let $\mathcal{C}(Z)$ denote the Calkin algebra associated with $\mathcal{B}\left(\ell^{2}(Z)\right)$. By modifying the proof of Lemma 1.6 and meeting some additional dense sets, we can assure that the restriction $\Phi_{Z}$ of $\Phi$ to $\mathcal{C}(Z)$ is an automorphism of $\mathcal{C}(Z)$.

Theorem 1 implies $\Phi_{Z}$ is inner and Lemma 3.3 implies $\Phi_{Z}$ is outer. This contradiction concludes the proof of Theorem 2 .

\section{Concluding remarks}

The existence of a nontrivial automorphism of $\mathcal{P}(\mathbb{N}) /$ Fin clearly implies the existence of a nontrivial automorphism of $\mathcal{P}(\kappa) /$ Fin for every infinite $\kappa$. Velickovic announced that it is possible to construct a nontrivial automorphism of $\mathcal{P}\left(\aleph_{2}\right) /$ Fin by other means (see [13, p. 13]) but the proof of this result is unfortunately not available. The situation with automorphisms of Calkin algebras is even less clear. There are no obvious implications between the existence of outer automorphisms of the Calkin algebra associated with Hilbert spaces of different densities. I don't even know whether it is relatively consistent with ZFC that the Calkin algebra associated with some nonseparable Hilbert space has an outer automorphism?

While $\mathcal{B}(H)$ has the unique nontrivial two-sided closed ideal if $H$ is separable, in the nonseparable case there are as many such ideals as there are infinite cardinals less or equal than the character density of $H$. Therefore there are several 'Calkin algebras' associated with a large Hilbert space $H$. The existence of outer automorphisms of these algebras will be investigated in a forthcoming joint paper with Ernest Schimmerling and Paul McKenney. 


\section{References}

1. B. Blackadar, Operator algebras, Encyclopaedia of Mathematical Sciences, vol. 122, SpringerVerlag, Berlin, 2006, Theory of $C^{*}$-algebras and von Neumann algebras, Operator Algebras and Non-commutative Geometry, III.

2. I. Farah, Analytic quotients: theory of liftings for quotients over analytic ideals on the integers, Memoirs of the American Mathematical Society, vol. 148, no. 702, 2000.

3. L Liftings of homomorphisms between quotient structures and Ulam stability, Logic Colloquium '98, Lecture notes in logic, vol. 13, A.K. Peters, 2000, pp. 173-196.

4. __ Rigidity conjectures, Logic Colloquium 2000, Lect. Notes Log., vol. 19, Assoc. Symbol. Logic, Urbana, IL, 2005, pp. 252-271.

5. __ All automorphisms of the Calkin algebra are inner, Annals of Mathematics 173 (2011), 619-661.

6. I. Farah and E. Wofsey, Set theory and operator algebras, Appalachian set theory 2006-2010 (James Cummings and Ernest Schimmerling, eds.), Mathematical logic, Ontos Verlag, to appear.

7. K. Kunen, Set theory: An introduction to independence proofs, North-Holland, 1980.

8. J.T. Moore, The proper forcing axiom, Proceedings of the ICM 2010, to appear.

9. G.K. Pedersen, Analysis now, Graduate Texts in Mathematics, vol. 118, Springer-Verlag, New York, 1989.

10. N.C. Phillips and N. Weaver, The Calkin algebra has outer automorphisms, Duke Math. Journal 139 (2007), 185-202.

11. S. Shelah, Proper forcing, Lecture Notes in Mathematics 940, Springer, 1982.

12. S. Todorcevic, Combinatorial dichotomies in set theory, Bull. Symb. Logic 17 (2011), 1-72.

13. B. Veličković, $O C A$ and automorphisms of $\mathcal{P}(\omega) /$ Fin, Top. Appl. 49 (1992), 1-13.

14. N. Weaver, Set theory and $C^{*}$-algebras, Bull. Symb. Logic 13 (2007), 1-20.

Department of Mathematics and Statistics, York University, 4700 Keele Street, North York, Ontario, Canada, M3J 1P3, and Matematicki Institut, Kneza Mihaila 34, Belgrade, SERBIA

$U R L:$ http://www.math.yorku.ca/ ifarah

E-mail address: ifarah@mathstat.yorku.ca 